\title{
Successful treatment of cervical and upper thoracic esophageal adenocarcinoma using induction chemotherapy followed by surgery: a case report
}

\section{Takeharu Imai}

Gifu University School of Medicine Graduate School of Medicine: Gifu Daigaku Igakubu Daigakuin Igakukei Kenkyuka

Yoshihiro Tanaka ( $\nabla$ yoshihirotana11@hotmail.com )

Gifu University School of Medicine Graduate School of Medicine: Gifu Daigaku Igakubu Daigakuin Igakukei Kenkyuka https://orcid.org/0000-0002-0300-7924

\section{Hidenori Ojio}

Gifu University School of Medicine Graduate School of Medicine: Gifu Daigaku Igakubu Daigakuin Igakukei Kenkyuka

\section{Yuta Sato}

Gifu University School of Medicine Graduate School of Medicine: Gifu Daigaku Igakubu Daigakuin Igakukei Kenkyuka

\section{Tomonari Suetsugu}

Gifu University School of Medicine Graduate School of Medicine: Gifu Daigaku Igakubu Daigakuin Igakukei Kenkyuka

\section{Masahiro Fukada}

Gifu University School of Medicine Graduate School of Medicine: Gifu Daigaku Igakubu Daigakuin Igakukei Kenkyuka

\section{Itaru Yasufuku}

Gifu University School of Medicine Graduate School of Medicine: Gifu Daigaku Igakubu Daigakuin Igakukei Kenkyuka

\section{Yoshinori Iwata}

Gifu University School of Medicine Graduate School of Medicine: Gifu Daigaku Igakubu Daigakuin Igakukei Kenkyuka

\section{Ryutaro Mori}

Gifu University School of Medicine Graduate School of Medicine: Gifu Daigaku Igakubu Daigakuin Igakukei Kenkyuka

\section{Hisashi Imai}

Gifu University School of Medicine Graduate School of Medicine: Gifu Daigaku Igakubu Daigakuin Igakukei Kenkyuka

\section{Takazumi Kato}


Gifu University School of Medicine Graduate School of Medicine: Gifu Daigaku Igakubu Daigakuin Igakukei Kenkyuka

\section{Naoki Okumura}

Gifu University School of Medicine Graduate School of Medicine: Gifu Daigaku Igakubu Daigakuin Igakukei Kenkyuka

\section{Nobuhisa Matsuhashi}

Gifu University School of Medicine Graduate School of Medicine: Gifu Daigaku Igakubu Daigakuin Igakukei Kenkyuka

\section{Takao Takahashi}

Gifu University School of Medicine Graduate School of Medicine: Gifu Daigaku Igakubu Daigakuin Igakukei Kenkyuka

\section{Manabu Futamura}

Gifu University School of Medicine Graduate School of Medicine: Gifu Daigaku Igakubu Daigakuin Igakukei Kenkyuka

\section{Kei Noguchi}

Department of Pathology, Gifu University Hospital

\section{Tatsuhiko Miyazaki}

Department of Pathology, Gifu University Hospital

\section{Kazuhiro Yoshida}

Gifu University School of Medicine Graduate School of Medicine: Gifu Daigaku Igakubu Daigakuin Igakukei Kenkyuka

\section{Case report}

Keywords: Cervical esophageal adenocarcinoma, Chemotherapy, Total laryngopharyngoesophagectomy

Posted Date: December 21st, 2020

DOl: https://doi.org/10.21203/rs.3.rs-131095/v1

License: (c) (i) This work is licensed under a Creative Commons Attribution 4.0 International License. Read Full License 


\section{Abstract}

\section{Background}

Cervical esophageal adenocarcinoma has a low incidence rate and its treatment involves various strategies. We report a patient with locally advanced cervical to upper esophageal adenocarcinoma, able to undergo induction chemotherapy and radical surgery. We report our case as a valuable clinical experience.

Case presentation

The patient complained of throat stuffiness. We found a poorly differentiated adenocarcinoma between the cervical esophagus and the upper thoracic esophagus. The primary lesion had infiltrated into the tracheal membrane and had metastatized into the cervical lymph nodes. The initial diagnosis was T4bN1M0 stage IVA. The lower edge of the tumor was close to the tracheal bifurcation, making it difficult to create a longitudinal tracheal foramen during surgery. Therefore, when biweekly-DCF therapy was performed as induction chemotherapy, the tumor shrank sufficiently. Furthermore, tumor infiltration into the tracheal membrane decreased, allowing us to perform total laryngopharyngoesophagectomy with three-field lymph node dissection and reconstruction using free jejunal grafts and subtotal stomach via a posterior mediastinum route and a permanent tracheal foramen as a radical surgery. The pathological diagnosis was T2/MP, N1, and the effect of chemotherapy was grade 2. The patient is followed-up regularly.

\section{Conclusions}

Cervical esophageal adenocarcinoma was rare, but technically reliable and safe oncologic surgery was possible after induction chemotherapy.

\section{Background}

Esophageal cancer is the eighth most common cancer worldwide and the sixth most common cause of death from cancer [1]. Based on histopathology, esophageal cancer is divided into squamous cell carcinoma (SCC) and adenocarcinoma. These two forms have different pathogenesis, epidemiology, tumor biology, and prognosis, requiring different therapeutic strategies [2]. Squamous cell carcinoma is the most common histology globally, while adenocarcinoma is the most common histology in Western countries [3]. The incidence rate of esophageal adenocarcinoma has been increasing worldwide [4]. However, the main tumor is located at the lower thoracic esophagus and esophagogastric junction (EGJ) because it is related to reflux-associated BE (Barrett's esophagus).

Cervical esophageal adenocarcinoma is extremely rare, with cervical squamous cell carcinoma accounting for $13.6 \%$ of esophageal SCC and cervical esophageal adenocarcinoma accounting for $0.3 \%$ of esophageal adenocarcinoma [2]. Therefore, no standard treatment exists for cervical adenocarcinoma. 
Various treatment strategies have been reported, especially for advanced cancer, including surgery alone, preoperative adjuvant chemotherapy plus surgery, surgery plus postoperative adjuvant chemotherapy, chemotherapy only, radiation therapy only, and chemoradiotherapy [5].

Our patient was a case of highly locally advanced cervical esophageal adenocarcinoma with tracheal infiltration, but induction chemotherapy was effective and radical resection was possible. Since cervical esophageal adenocarcinoma is rare, with no established treatment strategy, we report our case as a valuable clinical experience.

\section{Case Presentation}

A 55-years-old man, complaining of a feeling of throat stuffiness, consulted a nearby doctor, and was diagnosed with a tumor at the entrance of the esophagus by esophagogastroduodenoscopy (EGD). He was referred to our hospital for treatment. The patient's height was $165 \mathrm{~cm}$, with a body mass index of 21.0. He had no history of drinking and smoking. In our hospital, EGD showed a type2 tumor, $5 \mathrm{~cm}$ in diameter, located $20 \mathrm{~cm}$ from the incisors (Fig. 1a). Histopathological findings, of a biopsy taken, showed poorly differentiated adenocarcinoma with invasive growth, and the background mucosa showing glandular ducts consisting of a highly columnar epithelium with abundant mucus (considered to be ectopic gastric mucosa). Additionally, EGD showed no abnormal findings from the tumor to the EGJ, and the diagnosis was primary esophageal adenocarcinoma originating from the ectopic gastric mucosa. Contrast-enhanced CT showed swollen bilateral recurrent nerve lymph nodes and left supraclavicular lymph node, but no distant metastases. The primary lesion extending from the cervical esophagus to the upper thoracic esophagus was in contact with the tracheal membrane (Fig. 1b). Bronchoscopy showed no abnormal findings in the mucosal blood vessels and the tumor was not exposed, but the tracheal membrane was excluded and raised by the tumor (Fig. 2a). MRI detected a partially obscurity of the tracheal membrane, suggesting tracheal infiltration of the tumor (Fig. 2b).

Upper gastrointestinal series (UGI) revealed a $5 \mathrm{~cm}$-diameter tumor at the cervical and upper thoracic esophagus. The upper edge of the tumor was $2 \mathrm{~cm}$ oral from the upper sternum and its lower edge was $5 \mathrm{~cm}$ above the bifurcation of the trachea (Fig. 3a). PET-CT showed high FDG accumulation in the tumor, with SUVmax of 13.29 (Fig. 3b). Based on these findings, the initial diagnosis was cervical and upper thoracic adenocarcinoma, T4b/main bronchus, N1, M0 stage IVA, according to TNM classification in the UICC 8th edition. Due to the invasion of the tracheal membrane, it was possible to remove the tumor by total pharyngeal-laryngeal esophagectomy, including the trachea. However, because the significant distance between the lower edge of the tumor and tracheal bifurcation, the position of the mediastinal tracheal foramen was expected to be in the chest, which could significantly decrease the patient's quality of life (QOL). Therefore, first, the policy was to shrink the tumor by induction chemotherapy. The patient received two courses of chemotherapy, with biweekly DCF regimen: docetaxel $35 \mathrm{mg} / \mathrm{m} 2$ with cisplatin $40 \mathrm{mg} / \mathrm{m} 2$ on days 1 and 15 , and 5 -fluorouracil $400 \mathrm{mg} / \mathrm{m} 2$ on days $1-5$ and $15-19$ every 4 weeks. The treatment caused a Grade 4 neutropenia as an adverse event but with no sign of infection. After completion of the chemotherapy at $100 \%$ dose, EGD and enhanced CT showed tumor shrinkage, and the 
clinical effect was partial response (Fig. $1 \mathrm{~cd}$ ). Bronchoscopy showed improved tumor exclusion of the tracheal membrane (Fig. 2c). In addition, bronchoscopic ultrasound confirmed the absence of tumor infiltration in the tracheal membrane and MRI also confirmed a boundary between the tumor and the tracheomembrane (Fig. 2d). From these findings, we concluded that the infiltration of the tumor into the tracheal membrane had disappeared with chemotherapy. Furthermore, in UGI, the tumor shrank from the upper thoracic esophagus toward the cervical esophagus, and the distance between the lower edge of the tumor and the tracheal bifurcation was sufficient at $8 \mathrm{~cm}$ or more (Fig. 3c). PET-CT showed that FDG accumulation in the tumor disappeared (Fig. 3d). Therefore, the post-chemotherapy diagnosis was cyT3, cyN0, cyMO stage IIA (UICC8th), and a down stage was obtained. We planned to perform radical surgery (Fig. 3e).

Approximately 3 months after chemotherapy initiation, we performed total laryngopharyngoesophagectomy with three-field lymph node dissection and reconstruction using free jejunal grafts and subtotal stomach via a posterior mediastinum route and jejunostomy (Fig. 4a). The surgery was performed jointly with gastrointestinal surgery, head and neck surgery, and plastic surgery, and the operating time was 12 hours and 30 minutes, and the amount of bleeding was $660 \mathrm{ml}$. We performed thoracic esophagectomy in the left hemi-prone position using thoracoscopy. In the thoracic cavity, the bilateral bronchial arteries were preserved to maintain blood flow in the oral side of the trachea. The right bronchial artery diverged from the right subclavian artery (Fig. 4b). The tumor was detached from the tracheal membrane, the trachea was cut between the 5-6th tracheal cartilage, and a permanent tracheal foramen could be constructed at the upper sternal margin level (Fig. 4b,c). The thoracic duct was resected at the left venous angle of the neck. The thyroid gland was not resected, and the isthmus was dissected. During reconstruction, a free jejunum was placed between the mesopharynx and the subtotal stomach, and the jejunal artery and superior thyroid artery were anastomosed, and the jejunal vein and the total facial vein were anastomosed (Fig. 4d).

The resected specimen showed a $2 \mathrm{~cm}$ scar-like tumor on the mucosal surface of the cervical esophagus (Fig. 5ab). Histopathological findings confirmed a moderately and poorly differentiated adenocarcinoma, and the background mucosa showing ectopic gastric mucosa (Fig. $5 \mathrm{~cd}$ ). The pathological diagnosis was T2 MP, N1, and induction chemotherapy response was grade 2.

The ventilator was withdrawn on postoperative day (POD) 1, and the patient started to get out of bed. The drain in the right thoracic cavity was removed on POD 6 . The patient started swallowing training with jelly on POD 9, and eating orally on POD 14. A thrombus was observed in the right femoral vein to the inferior vena cava on POD 15, which was controlled by continuous intravenous heparin injection and oral warfarin. After confirming that the thrombus had shrunk, the patient was switched to an oral anticoagulant. The patient's condition improved thereafter. He practiced bone conduction vocalization and was discharged on POD 35. Three months after surgery, we followed-up on our patient in our outpatient clinic without adjuvant chemotherapy.

\section{Discussion And Conclusions}


Squamous cell carcinomas (SCCs) and adenocarcinomas are two types of esophageal cancer. In Japan and the East, many SCCs are reported in the upper and middle thoracic esophagus, while in the West, there are many adenocarcinomas reported in the lower thoracic esophagus, up to the esophagogastric junction (EGJ) [4]. BE is risk factor for esophageal adenocarcinoma and this histological type of lower thoracic esophagus and EGJ has increased worldwide [6, 7]. However, adenocarcinoma of the cervical and upper thoracic esophagus is very rare. Moreover, it is often found in advanced cancer, and its prognosis is poor [4]. Previously, we reported a patient with adenocarcinoma in the upper thoracic esophagus who underwent surgery [8]. In our department, among all patients who underwent esophageal cancer surgery in the last 10 years, two patients $(0.6 \%)$ including this case had adenocarcinoma of the cervical to upper thoracic esophagus, which is also rare.

Since cervical or upper thoracic esophageal adenocarcinoma is rare, it is important to diagnose primary esophageal adenocarcinoma. In Japan, there is a definition of primary esophageal adenocarcinoma by Komei Nakayama, (a) tumors derived from the esophageal mucosa or mucous glands, (b) a healthy squamous epithelium on the anal side or a tumor located entirely on the oral side of the cardia ring, (c) no cancerous changes have occurred in other organs [9]. Most primary adenocarcinomas of the esophagus are derived from BE, ectopic gastric mucosa (EGM), or the esophageal glands [10-12]. In our case, ectopic gastric mucosa was found in the background mucosa of the tumor in the histopathological findings of biopsy before chemotherapy and in the resected specimen, and no abnormal findings were found between the tumor and EGJ. Furthermore, no neoplastic lesions were found in other organs, and the diagnosis was primary cervical to upper thoracic esophageal adenocarcinoma originating from EGM. The relationship between EGM and adenocarcinoma is still unclear, and further studies are needed to investigate their relationship in a larger number of patients [13]. Nomura et al. reviewed the literature on adenocarcinoma arising from ectopic gastric mucosa in the cervical or upper thoracic esophagus [13]. According to their review, majority of the cases were well- or moderately differentiated adenocarcinomas, and only $24.0 \%$ of cases were poorly differentiated. Our patient had poorly differentiated adenocarcinoma.

The treatment of cervical esophageal adenocarcinoma is varied. Nomura et al. also reported that treatment strategies depended on the tumor stage: open chest surgery with or without pre/post adjuvant therapy in 37 patients, endoscopic therapy in 8, chemoradiotherapy in 2, and radiation alone in 2 [13]. Moreover, the prognosis for advanced cancer was poor because there was only one survivor of T3-4 [13]. In our department, we usually perform chemoradiotherapy (DF + RT) for unresectable, locally advanced esophageal SCC and achieve good results [14]. However, we usually do not actively administer CRT to esophageal adenocarcinoma, including EGJ. Esophageal adenocarcinomas are less radiosensitive than esophageal squamous cell carcinomas, and all patients who are operable for esophageal adenocarcinoma that is potentially curable should be considered for neoadjuvant chemotherapy or chemoradiotherapy followed by surgery [15]. Since our case was esophageal AC, we considered it important to bring it to radical resection by induction treatment when aiming for a good prognosis. Patients who received induction chemotherapy experienced a trend toward superior survival for not only surgery but also chemoradiotherapy [5]. It was thought that tumor shrinkage due to induction 
chemotherapy reduces the amount of radiation therapy, and controls micrometastasis. Definitive chemoradiation (DCRT) has been adopted in the treatment of upper esophageal cancers and has become the standard modality recommended by most experts $[5,16]$. However, it is controversial, especially for cervical esophageal adenocarcinoma, and local recurrence rates after definitive chemoradiotherapy are high (40-75\%) [3]. Moreover, a higher mortality and morbidity rate, including anastomotic leak and pulmonary complications, was reported after salvage surgery $[3,17]$. Therefore, we considered that salvage surgery and surgery for recurrent cases after DCRT were technically extremely difficult. From the perspective of prolonging survival, we prioritize surgery for patients who can undergo radical resection.

According to randomized trials, including the OEO2 and MAGIC trial, standard chemotherapy is platinumfluoropyrimidine-based [15]. We usually perform biweekly-DCF (Bi-DCF) chemotherapy for esophageal SCC and EGJ adenocarcinoma with esophageal infiltration, whether it is induction chemotherapy for unresectable or NAC for resectable tumors [18]. We have experienced and reported EGJ adenocarcinoma with pathological CR obtained by radical resection after Bi-DCF chemotherapy [19]. Therefore, we selected Bi-DCF chemotherapy because the regimen was considered effective for rare cases of cervical esophageal adenocarcinoma. As a result, as we aimed, the tumor shrank by induction chemotherapy, the pathological effect of chemotherapy-grade 2 was obtained, and radical resection was possible. Historically, most patients with upper thoracic esophageal cancer were treated by surgery, including pharyngo-laryngo-esophagectomy (PLE) and gastric pull-up. In Japan, we have "Esophageal Cancer Practice Guidelines" for esophageal cancer treatment [20]. In the treatment of cervical esophageal carcinoma, this guideline recommended that we should select chemoradiotherapy or surgery, including laryngectomy, with due consideration given to quality of life. Of course, we chose PLE with the most favorable prognosis after fully explaining that radical resection would cause loss of voice and reduced QOL for this patient.

There are some reports but no clear evidence of success of postoperative adjuvant therapy after radical resection after NAC, as in our case [21-23]. For esophageal SCC, we usually recommend postoperative adjuvant chemotherapy for pathological lymph node metastasis-positive cases because the prognosis of pathological node-positive cases was much worse than that of negative cases. Naoki reported that surgery with pre/postoperative radiation is advisable for the local control of advanced tumors, and surgery with pre/postoperative chemoradiotherapy can prevent both local recurrence and distant metastasis [24]. In our case, the patient was positive for pathological lymph node metastases, but we had undergone maximum surgery followed by NAC, and since the tumor was a rare adenocarcinoma, we decided to follow up closely without adjuvant therapy.

Our patient was a case of highly locally advanced cervical esophageal adenocarcinoma with tracheal infiltration, but induction chemotherapy was effective and radical resection was possible. Cervical esophageal adenocarcinoma was rare, but technically reliable and safe oncologic surgery was possible after induction chemotherapy.

\section{Abbreviations}


SCC: Squamous cell carcinoma; EGJ: Esophagogastric junction; BE: Barrett's esophagus; EGD: esophagogastroduodenoscopy; CT: Computed tomography; MRI: Magnetic Resonance Imaging; UGI: Upper gastrointestinal series; PET: Positron-emission tomography; QOL: Quality of life; POD: Postoperative day; EGM: Ectopic gastric mucosa; DCRT: Definitive chemoradiation therapy; Bi-DCF: Biweekly-DCF; PLE: pharyngo-laryngo-esophagectomy; NAC: Neoadjuvant chemotherapy

\section{Declarations}

\section{Authors' contributions}

$\mathrm{TI}, \mathrm{YT}, \mathrm{HO}, \mathrm{YS}$ and $\mathrm{KY}$ managed the patient. KN and TM performed the histopathological examination. TI and $\mathrm{YT}$ wrote the manuscript and provided the original pictures. All the other authors reviewed the manuscript. All authors approved the final manuscript.

\section{Funding}

The authors have no funding related to this article to declare.

\section{Conflicts of Interest}

Dr. Yoshida reports receipt of grants, personal fees and non-financial support from EA Pharma Co., Ltd., Sanofi, Yakult Honsha Co., Ltd., Chugai Pharmaceutical Co., Ltd., Taiho Pharmaceutical Co., Ltd., Takeda Pharmaceutical Co., Ltd., Eli Lilly Japan K.K., Daiichi Sankyo Co., Ltd., Ono Pharmaceutical Co., Ltd., Merck Serono Co., Ltd., and Novartis Pharma K.K.; and grants from Kyowa Hakko Kirin Co., Ltd. outside of the submitted work. Dr. Tanaka reports receipt of grants from Daiichi Sankyo Co., Ltd. outside of the submitted work. Other authors have no conflict of interest to disclose. The funding source had no role in the design, practice, or analysis of this study.

\section{Availability of data and materials}

All data generated or analyzed during this study are included in this published article.

\section{Ethics approval and consent participate}

Not applicable.

\section{Consent for publication}

Informed consent was obtained from the patient for the publication of this case report and any accompanying images.

\section{Competing interests}

The authors have no competing interests related to this article to declare. 


\section{Acknowledgements}

We thank the department of head and neck surgery and department of plastic surgery at Gifu University Hospital for treating us together. This case report did not require ethical approval. The patient gave permission for the publication of this case report. We would like to thank Editage (www.editage.com) for English language editing.

\section{References}

1. Yamaji T, Tsugane S. Epidemiology of esophageal squamous cell carcinoma. In: Ando N, (ed.), Esophageal squamous cell carcinoma. Springer Nature Singapore Pte Ltd; 2020. p. 1-13.

2. Rudiger Siewert and Katja Ott. Are squamous and adenocarcinomas of the esophagus the same disease? Semin Radiat Oncol. 2007;17:38-44.

3. Ilson DH. Adenocarcinoma of the esophagus: controversies and consensus. Chin Clin Oncol. 2017;6(5):52.

4. Rubenstein JH, Shaheen NJ. Epidemiology, diagnosis, and management of esophageal adenocarcinoma. Gastroenterology. 2015;149(2):302-17.e1

5. Esmati E, Maddah Safaei A, Ghalehtaki R, Mousavi N, Saraee E, Shirouei S, et al. Outcomes of definitive chemoradiotherapy for cervical and upper thoracic esophageal cancers: a single-Institution Experience of a rare cancer. J Gastrointest Canc. 2019;50(3):380-5.

6. Chung JW, Lee GH, Choi KS, Kim DH, Jung KW, Song HJ, et al. Unchanging trend of esophagogastric junction adenocarcinoma in Korea: experience at a single institution based on Siewert's classificatio Dis Esophagus. 2009;22(8):676-81.

7. Coleman HG, Xie SH, Lagergren J. The epidemiology of esophageal adenocarcinoma. Gastroenterology. 2018;154(2):390-405.

8. Komori S, Osada S, Tanaka Y, Takahashi T, Nagao N, Yamaguchi K, et al. A case of esophageal adenocarcinoma arising from the ectopic gastric mucosa in the thoracic esophagus. Rare Tumors. 2010;2(1):e5. https://doi.org/10.4081/rt.2010.e5

9. Yasunaga A, Matsubara T, Maki A, Kinoshita I, Nisi M, Kajitani T, et al. A case report of primary multiple esophageal adenocarcinoma. Jpn J Gastroenterological Surg. 1989;22:2429-32.

10. Takebayashi K, Yamamoto H, Murata S, Ishida M, Yamaguchi T, Kojima M, et al. Cervical esophageal adenocarcinoma originating from the esophageal gland. Journal of Japanese College of Surgeons. 2014;39:187-92.

11. litaka D, Fujiwara H, Shiozaki A, Kubota T, Ando T, Murayama Y, et al. Double primary cancer of the esophagus consisting of ectopic gastric mucosa-derived adenocarcinoma and squamous cell carcinoma: a first case report. Esophagus. 2011;8(4):303-9.

12. Endoh Y, Miyawaki M, Tamura G, Watanabe H, Motoyama T. Esophageal adenocarcinoma that probably originated in the esophageal gland duct: a case report. Pathol Int. 1999;49(2):156-59. 
13. Nomura K, lizuka T, Inoshita N, Kuribayashi Y, Toba T, Yamada A, et al. Adenocarcinoma of the cervical esophagus arising from ectopic gastric mucosa: report of two cases and review of the literature. Clin J Gastroenterol. 2015;8(6):367-76.

14. Fukada $\mathrm{M}$, Tanaka $\mathrm{Y}$, Imai T, Matsui S, Imai $\mathrm{H}$, Matsuhashi $\mathrm{N}$ et al. Efficacy and safety of neoadjuvant chemoradiotherapy with docetaxel and 5-fluorouracil followed by esophagectomy to treat advanced esophageal cancer. Jpn J Gastroenterol Surg. 2019;52(12):753-63.

15. Lagergren J, Smyth E, Cunningham D, Lagergren P. Oesophageal cancer. Lancet. 2017;390(10110):2383-96.

16. Cooper JS, Guo MD, Herskovic A, Macdonald JS, Martenson JA, Al-Sarraf M, et al. Chemoradiotherapy of locally advanced esophageal cancer: long-term follow up of a prospective randomized trial (RTOG85-01). JAMA. 1999;281:462-7.

17. Markar SR, Karthikesalingam A, Penna M, Low DE. Assessment of short-term clinical outcomes following salvage esophagectomy for the treatment of esophageal malignancy: systematic review and pooled analysis. Ann Surg Oncol. 2014;21(3):922-31.

18. Tanaka Y, Yoshida K, Yamada A, Tanahashi T, Okumura N, Matsuhashi N, et al. Phase II trial of biweekly docetaxel, cisplatin, and 5-fluorouracil chemotherapy for advanced esophageal squamous cell carcinoma. Cancer Chemother Pharmacol. 2016;77(6):1143-52.

19. Suetsugu T, Tanaka Y, Banno S, Imai T, Yamaguchi K, Yoshida K. A case of pathologic complete response with neoadjuvant biweekly-DCF therapy and following laparoscopic transhiatal extended total gastrectomy for advanced esophagogastric junction adenocarcinoma. Nihon Rinsho Geka Gakkai Zasshi (J Jpn Surg Assoc). 2019;80(4):707-13.

20. Kitagawa Y, Uno T, Oyama T, Kato K, Kato H, Kawakubo H, et al. Esophageal cancer practice guidelines 2017 edited by the Japan esophageal society: Part 2. Esophagus. 2019;16(1):25-43.

21. Drake J, Tauer K, Portnoy D, Weksler B. Adjuvant chemotherapy is associated with improved survival in patients with nodal metastases after neoadjuvant therapy and esophagectomy. $\mathrm{J}$ Thorac Dis. 2019;11(6):2546-54.

22. Semenkovich TR, Subramanian M, Yan Y, Hofstetter WL, Correa AM, Cassivi SD, et al. Adjuvant therapy for node-positive esophageal cancer after induction and surgery: A multisite study. Ann Thorac Surg. 2019;108(3):828-36.

23. Yan Wanpu, Zhao Peiliang, Fu H, Lin Y, Li Z, Dai L, et al. Survival after induction chemotherapy and esophagectomy is not improved by adjuvant chemotherapy. Ann Thorac Surg. 2019;108(5):1505-13.

24. Akanuma N, Hoshino I, Akutsu Y, Shuto K, Shiratori T, Kono T, et al. Primary esophageal adenocarcinoma arising from heterotopic gastric mucosa: report of a case. Surg Today. 2013;43:446-51.

\section{Figures}



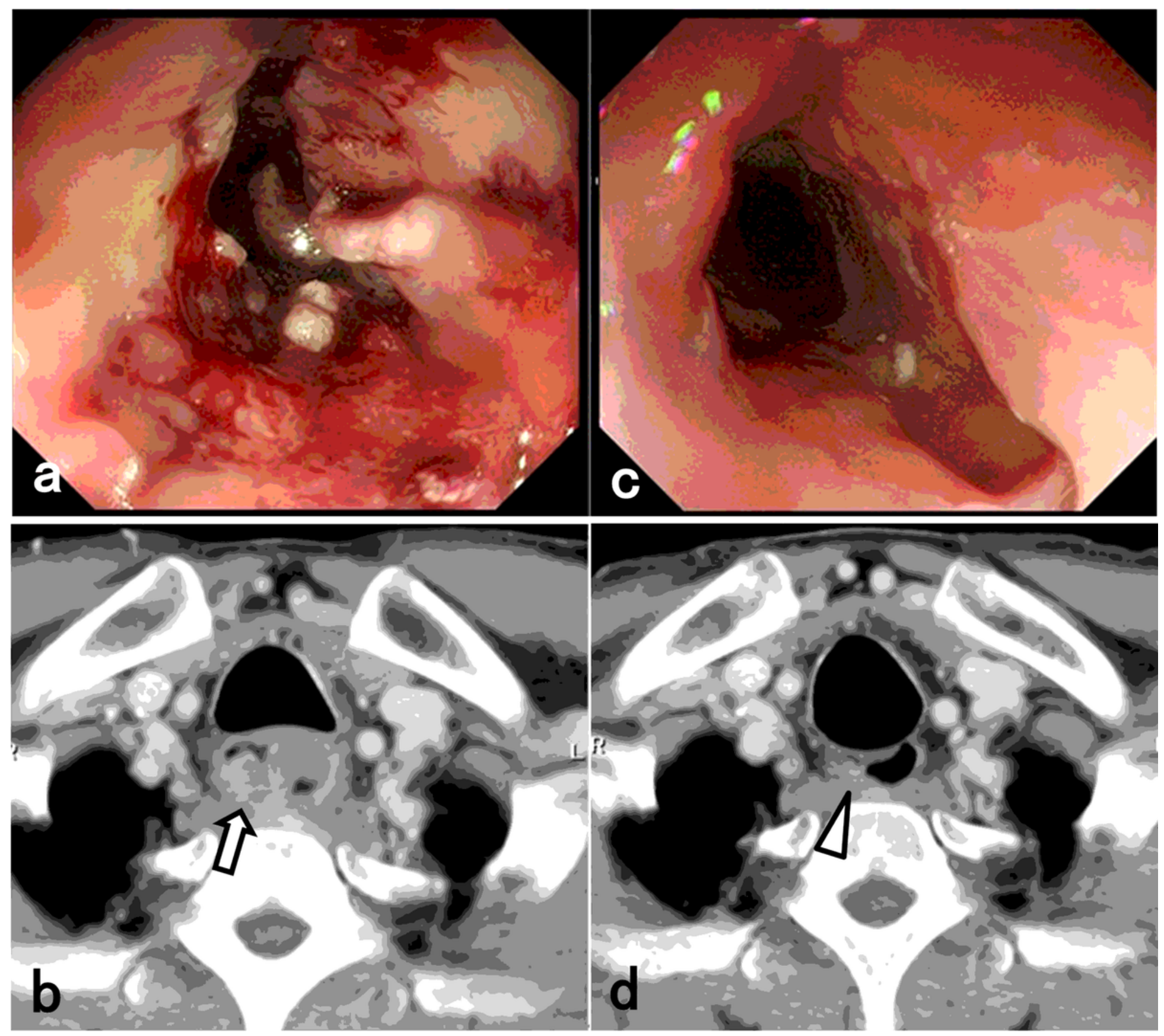

Figure 1

EGD and Contrast enhanced CT findings (a) EGD showed circumferential type 3 tumor at cervical to upper thoracic esophagus. (b) Enhanced CT showed thickened wall of the cervical esophagus (arrow). (c) Tumor decrease in size after induction chemotherapy. (d) Thickened wall improved after chemotherapy (arrowhead). 


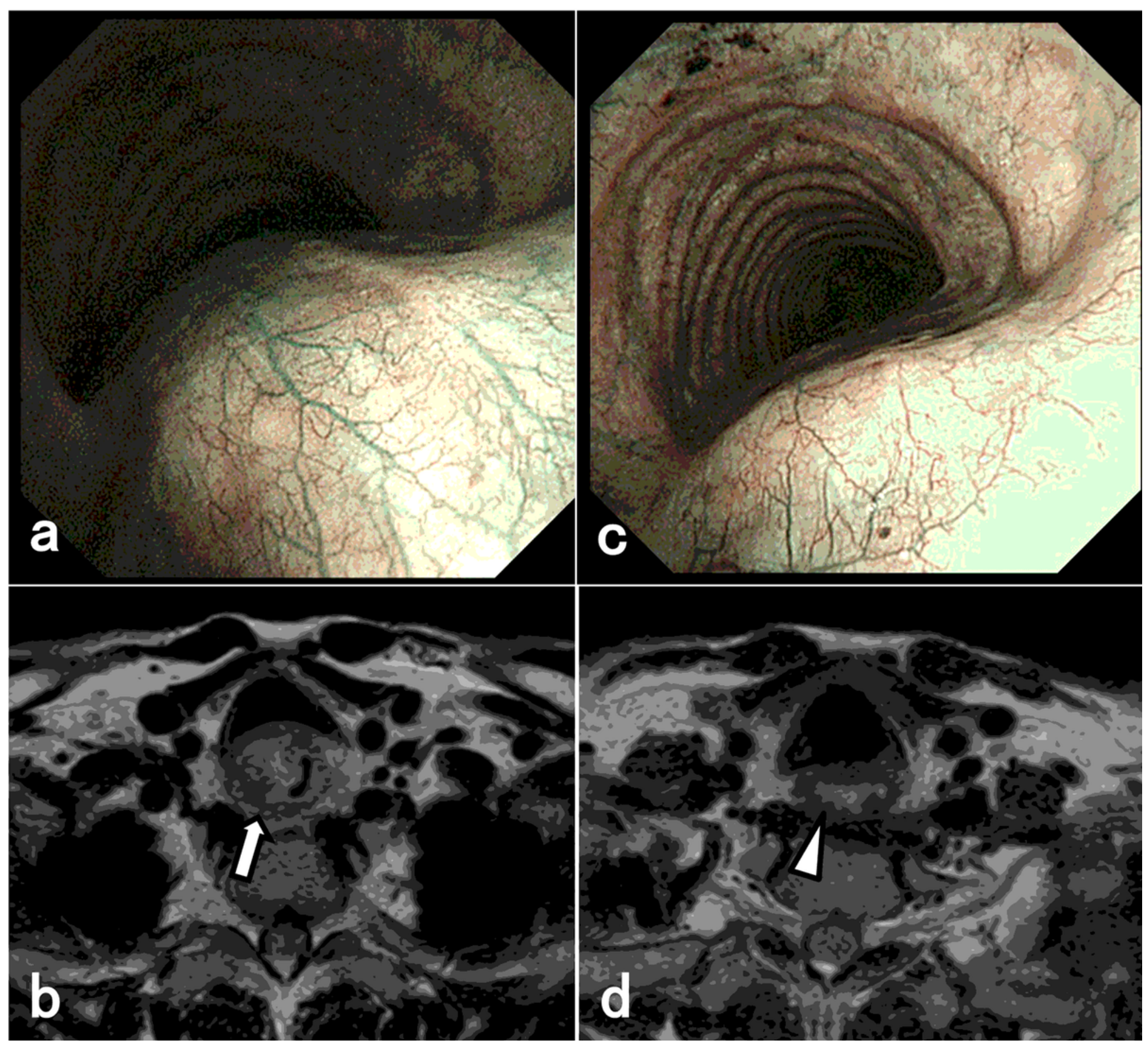

Figure 2

Bronchoscopy and MRI findings (a) Bronchoscopy showed the tracheal membrane raising by the tumor. (b) MRI showed tracheal membrane infiltration of the tumor (arrow). (c) Trachea membrane raising by the tumor improved. (d) The tumor shrank and its infiltration into the trachea membrane improved (arrowhead). 

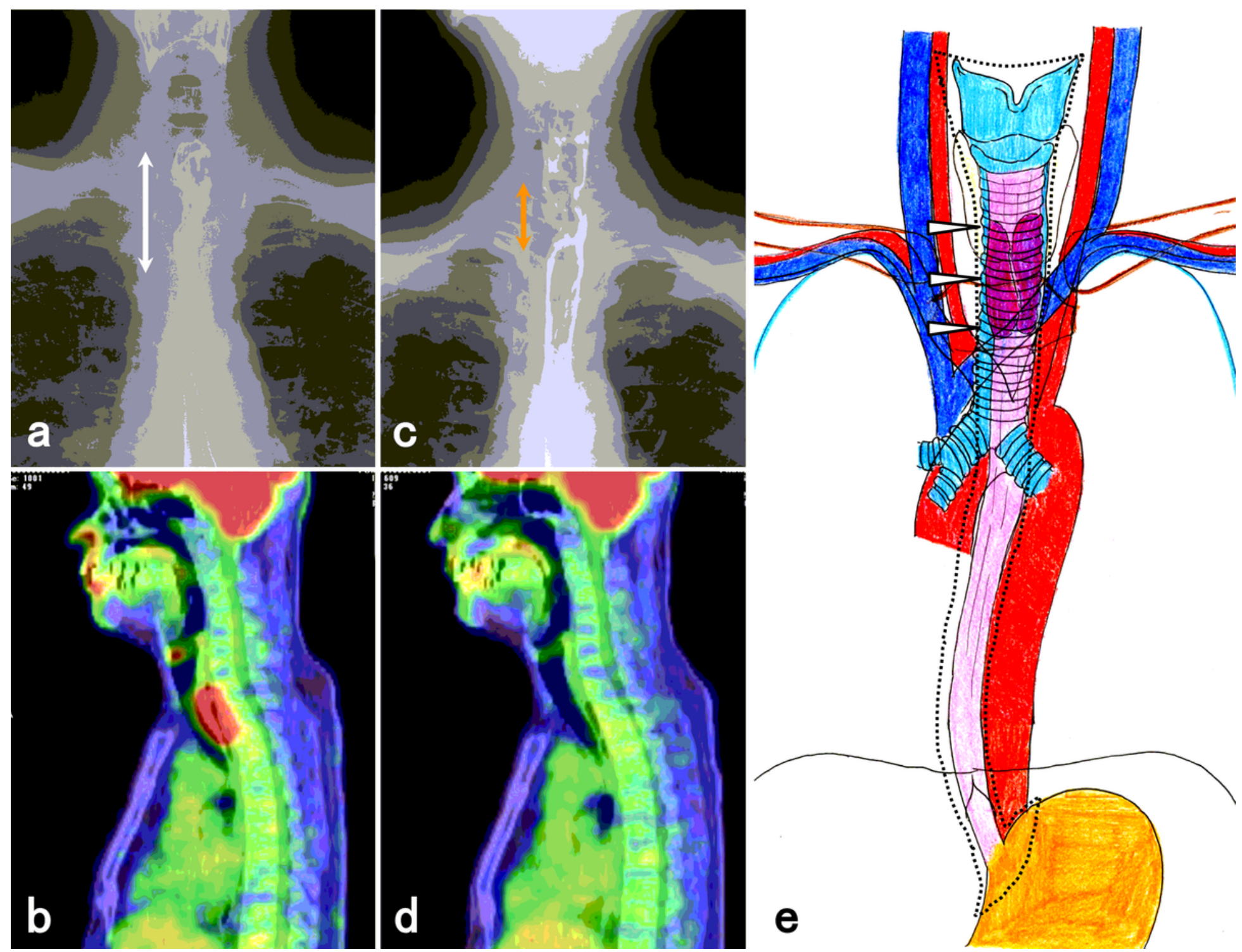

\section{Figure 3}

UGI and PET-CT findings (a) UGI showed the tumor at cervical and upper thoracic esophagus, which was $60 \mathrm{~mm}$ in diameter (arrow). (b) PET-CT showed high FDG accumulation of tumor at cervical and upper thoracic esophagus. (c) The tumor shrank from the upper thoracic esophagus toward the cervical esophagus after induction chemotherapy (orange arrow). (d) FDG accumulation in the tumor disappeared after chemotherapy. (e) Preoperative schema: the tumor (arrowhead) and the extent of resection (dotline). 


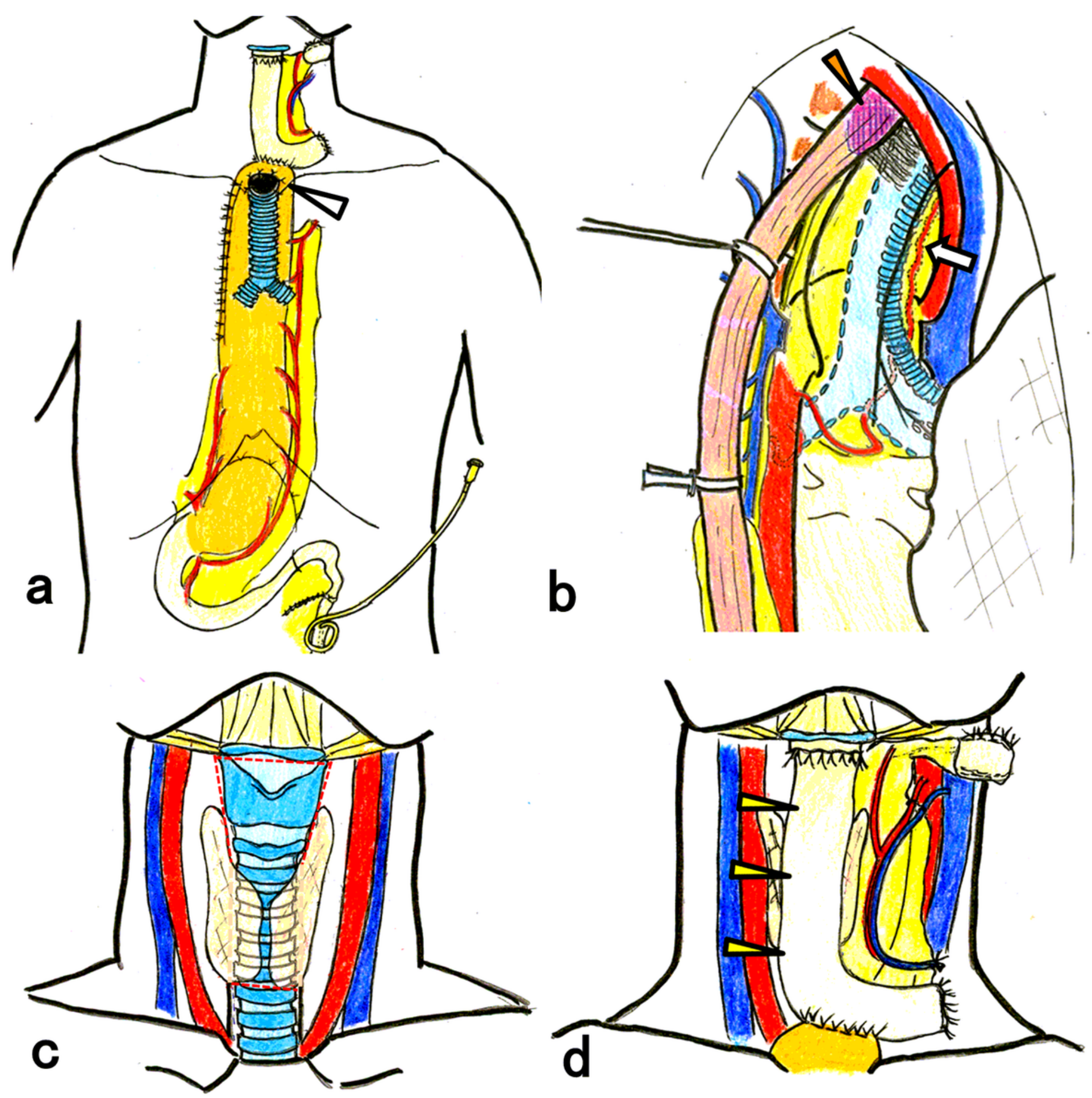

Figure 4

Operation findings (a) Total laryngopharyngoesophagectomy with three-field lymph node dissection and reconstruction using free jejunal grafts and sub-total stomach via a posterior mediastinum route, a permanent tracheal foramen (arrowhead) and jejunostomy were performed. (b) The bilateral bronchial arteries were preserved to maintain blood flow in the oral side of the trachea. The right bronchial artery diverged from the right subclavian artery (arrow). The tumor was detached from the tracheal membrane (orange arrowhead). (c) The trachea was cut between the 5-6th tracheal cartilage, and a permanent 
tracheal foramen was constructed at the upper sternal margin level (dot-line). (d) A free jejunum was placed between the mesopharynx and the subtotal stomach (yellow arrow).
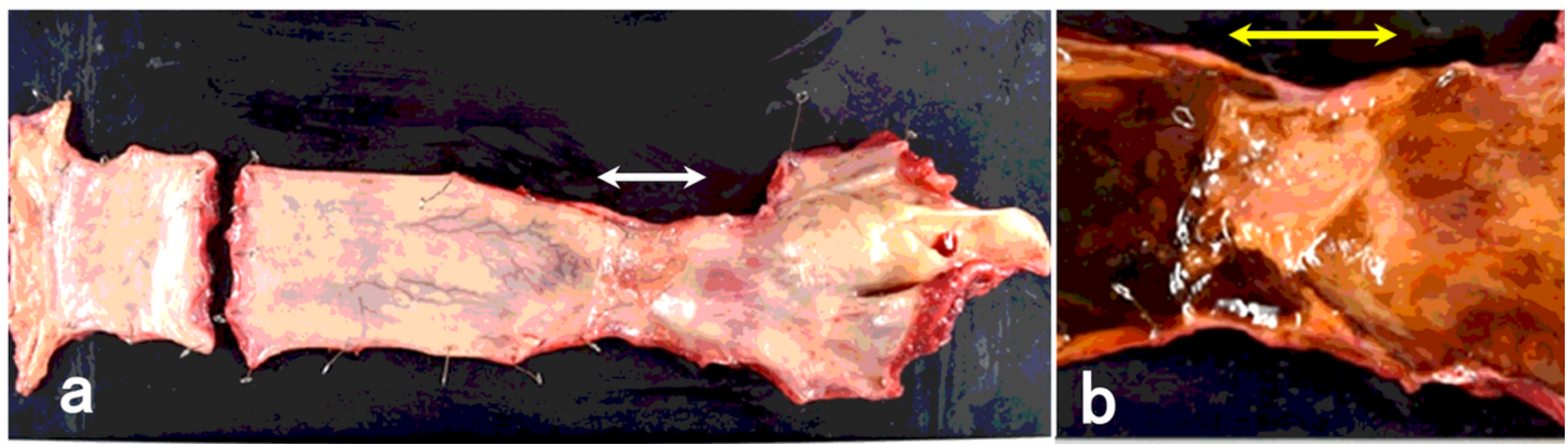

$\begin{array}{lllllllllllllllllllllllllllllllll}15 & 16 & 17 & 18 & 19 & 20 & 21 & 22 & 23 & 24 & 25 & 26 & 27 & 28 & 29 & 30 & 31 & 32 & 33 & 34 & 35 & 36 & 37 & 38 & 39 & 40 & 41 & 42 & 43 & 44 & 45 & 46 & 47\end{array}$
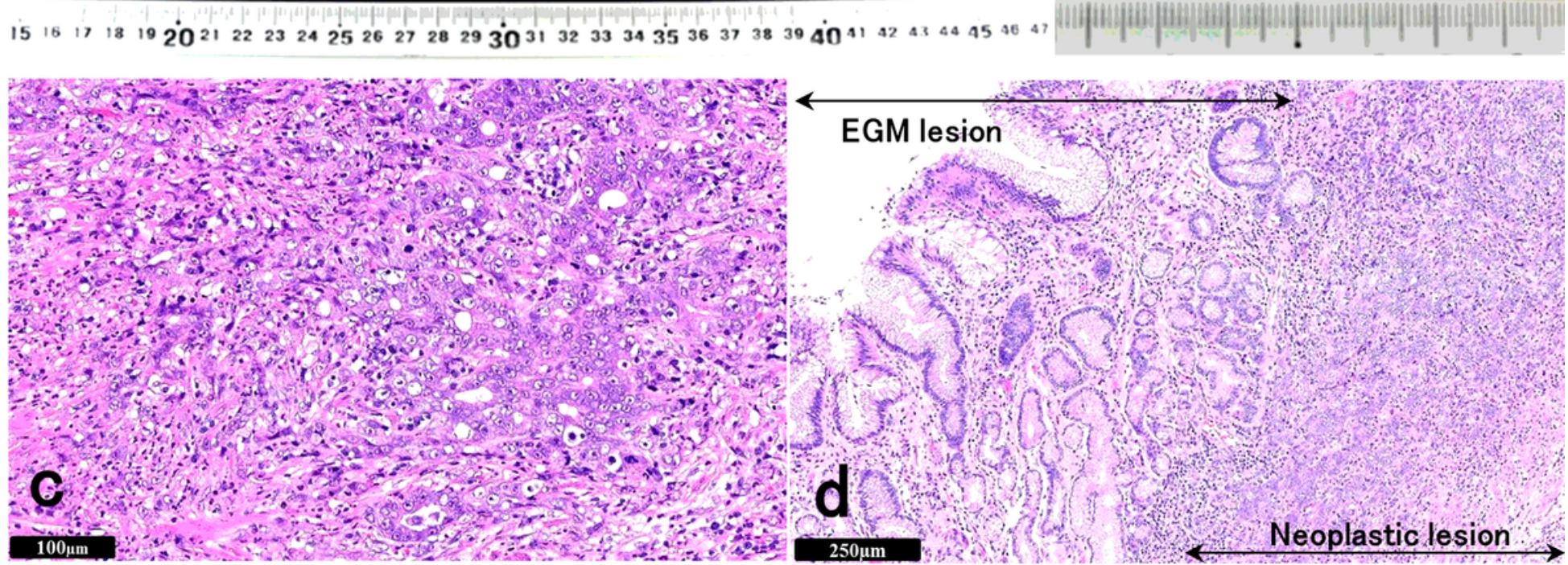

\section{Figure 5}

Resected specimen and histopathological findings (a) The resected specimen showed a $2 \mathrm{~cm}$ scar-like tumor on the cervical esophagus (arrow). (b) Tumor was not stained with lugol (yellow arrow). (c) Histopathological findings were poorly and moderately differentiated adenocarcinoma. (d) The background mucosa adjacent to the adenocarcinoma cells showed ectopic gastric mucosa (EGM). 